\title{
The Shape and Stability of Small Liquid Drops on Fibers
}

\author{
G. McHale ${ }^{1 *}$, M.I. Newton ${ }^{1}$ and B.J. Carroll ${ }^{2}$ \\ 1 Department of Chemistry and Physics, The Nottingham Trent University, Clifton Lane, Nottingham NG1 1 8NS - UK \\ 2 Unilever Research Laboratory, Quarry Road East, Bebington, Merseyside L63 3JW - UK \\ e-mail: glen.mchale@ntu.ac.uk \\ ${ }^{*}$ Corresponding author
}

Résumé — Forme et stabilité de gouttelettes de liquide sur des fibres — La forme d'une petite goutte de liquide sur une fibre de petit diamètre peut être décrite soit par une allure en tonneau axé symétrique, soit par une allure en perle (cloche). Les expériences montrent que lorsque le volume réduit, défini par le rapport entre le volume de la goutte et le rayon de la fibre, est grand, la configuration préférée est celle en tonneau. Lorsque le volume réduit est petit, une transition se produit vers la forme en perle. Le volume correspondant à cette transition dépend de l'angle de contact d'équilibre.

Dans cet article, nous considérons la solution connue de l'équation de Laplace pour la forme en tonneau. Nous étudions le lien entre le profil, le point d'inflexion dans le profil et la stabilité de la goutte. Dans le cas de la forme en cloche, il n'existe pas de solution connue de l'équation de Laplace. Nous développons ici une approche en éléments finis pour déterminer le profil d'une goutte sur une fibre et présentons des résultats numériques pour la forme en cloche. Les énergies de surface pour ces deux types de goutte sur une fibre sont calculées pour plusieurs volumes de goutte et angles de contact. Les implications correspondantes pour la stabilité de ces gouttes sont alors discutées.

Mots-clés : fibre, angle de contact, mouillage, stabilité, forme de goutte.

\begin{abstract}
The Shape and Stability of Small Liquid Drops on Fibers - The shape of a small liquid drop on a small diameter fiber may be either an axisymmetric barrel shape or it may be a nonaxisymmetric clam-shell shape. Experiments show that when the reduced volume, given by the volume of droplet divided by the fiber radius, is large the barrel shape is the preferred conformation, but that as the volume reduces a transition to a clam-shell (pearl) shape occurs. The volume at which this stability transition occurs depends upon the equilibrium contact angle.

In this work we review the known solution to Laplace's equation for the barrel shape and consider the link between the profile, the inflexion in the profile and the stability of the droplet. No known solution of Laplace's equation exists for the clam-shell shape droplet. We therefore consider a finite element approach to determining the possible shapes of a droplet on a fiber and give numerical results for the clam-shell profile. The surface free energies for the two types of droplet conformation on a fiber are computed for several droplet volumes and equilibrium contact angles and the implications of this for droplet stability are discussed.

Keywords: fiber, contact angle, wetting, stability, barrel, clam, pearl.
\end{abstract}




\section{INTRODUCTION}

In equilibrium a small droplet placed on a flat solid surface adopts a spherical cap shape with an equilibrium contact angle, $\theta_{e}$, given by Young's equation $\cos \theta_{e}=\left(\gamma_{S V}-\gamma_{S L}\right) /$ $\gamma_{L V}$, where $\gamma_{S V}, \gamma_{S L}$ and $\gamma_{L V}$ are the solid-vapor, solid-liquid and liquid-vapor interfacial tensions (Adamson and Gast, 1997). Thus information about the solid-liquid interaction can be gained by measurement of the contact angle provided the surface is one that is only partially wet by the liquid. In the case that the spreading power, $S=\gamma_{S V}-\left(\gamma_{S L}+\gamma_{L V}\right)$, is positive no equilibrium contact angle exists and the liquid spreads to form a thin film (de Gennes, 1985; Léger and Joanny, 1992). Chemically altering the solid surface will modify the interfacial tensions and can be used to control wetting. However, wetting of a flat surface can also be modified by control of the local geometry of the solid surface. Wenzel (1936) argued that surface roughness would alter the equilibrium contact angle. Richard and Quéré (1999) have recently demonstrated experimentally that modifying the local geometry, together with the surface chemistry, can enable control of the equilibrium wetting.

The influence of the geometry of a surface on its wetting properties is not restricted to ideas of local geometry as used in rough or patterned surfaces. A common shape of material is that of a fiber, i.e. a solid cylinder. The coating and wetting of fibers is important to many industries (Quéré, 1999). However due to the global geometry of the fiber shape, the wetting of a material in the form of a fiber is quite different to that of the same material in the form of a flat surface (McHale et al., 1997). A small droplet placed on a fiber can adopt one of two conformations (Figs. 1a and $1 \mathrm{~b}$ ): an axisymmetric barrel shape or a non-axisymmetric clam-shell shape (also known as a "pearl"). For larger volumes and smaller contact angles a barrel shape is preferred over the clam-shell shape. In detergency, the removal of oily soil in the form of a barrel is more difficult as it involves a larger solid-liquid interfacial area. Reducing the volume of a barrel shape leads to a stability transition resulting in the clam-shell shape. This is known as the roll-up process (Adam, 1937); a sequence of images showing this transition during a solubilization experiment is given by Carroll (Fig. 2 of Carroll, 1986). The fiber shape also results in other differences compared to the wetting of a flat surface. A vanishing contact angle, as defined by Young's equation, will on a flat surface give a film, but on a fiber it is possible that a macroscopic barrel shape will still exist (McHale et al., 1997; Carroll, 1976). A vanishing contact angle does not guarantee film formation on a fiber. The condition for film formation is no longer that the spreading power should simply be positive, but that it should be greater than some critical value (Brochard-Wyart et al., 1990; Brochard, 1986). In addition, a barrel-shape profile also involves a point of maximum slope, an inflexion, that can be quite different from the contact angle and which can occur close to the contact line. This can make it difficult to accurately measure the contact angle (McHale et al., 1997, 1999).

In this work we consider the influence of the fiber's global geometry on its wetting. In particular, we focus on the roll-up transition from a barrel shape to a clam-shell shape. The solution for the profile of a barrel is discussed in relation to minimizing the Laplace excess pressure. The consequences of this for the stability diagram are indicated and it is conjectured that an inflexion in the profile of a barrel shape is required for the droplet to be stable. As no known solution exists for the profile of a clam-shell shape, a finite element approach is developed. Preliminary results are presented giving the surface profiles and surface free energies for barrel shapes and clam-shell shapes of identical volume and contact angle.

\section{GLOBAL GEOMETRY VERSUS SURFACE CHEMISTRY}

On a flat surface a vanishing equilibrium contact angle indicates film formation. The energy per unit area for a vapor directly in contact with a solid is $\gamma_{S V}$. If some of the vapor now condenses into an infinitely thin film intercalated between the solid and the bulk vapor the energy will become $\left(\gamma_{S L}+\gamma_{L V}\right)$ due to the two interfacial areas. This latter situation is energetically preferred when $S=\gamma_{S V}-\left(\gamma_{S L}+\gamma_{L V}\right)$ is positive; $S$ is the spreading power. Rearranging shows that if $\left(\gamma_{S V}-\gamma_{S L}\right) / \gamma_{L V}>1$ a film forms. However, the left-hand side of this inequality is simply Young's equation for $\cos \theta_{e}$ and so film formation is preferred once the equilibrium contact angle vanishes; indeed it is no longer correct to refer to equilibrium contact angles. This concept of film formation
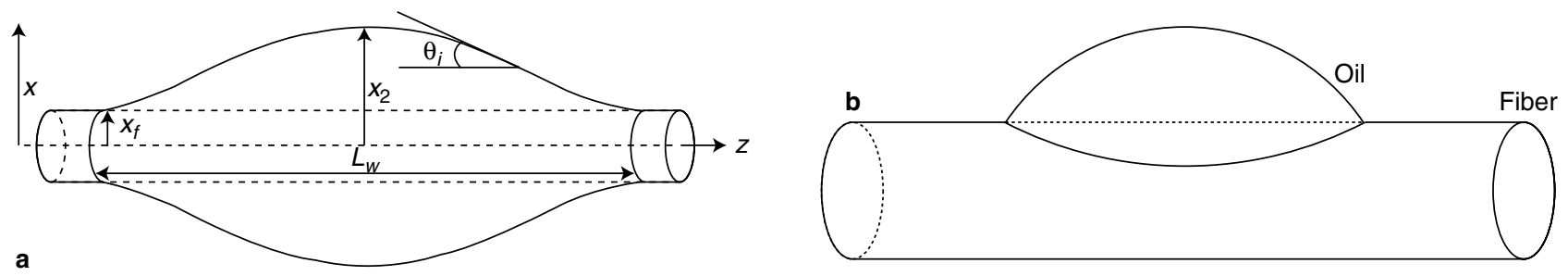

Figure 1

The two preferred conformations on a fiber are (a) the barrel shape, and (b) the clam-shell (pearl) shape. 
can be related to the Laplace excess pressure across the liquid-vapor surface of a small drop of liquid resting on a flat solid surface. The Laplace pressure is given by:

$$
\Delta P=\gamma_{L V}\left(\frac{1}{R_{1}}+\frac{1}{R_{2}}\right)
$$

where $R_{1}$ and $R_{2}$ are the two principal radii of curvature at a point in the surface. If we now reduce the contact angle, but maintain the spherical cap shape, both radii of curvature will increase; this can be seen by considering the radii at either the apex of the droplet or the contact line. A film will form because the curvature will vanish at all points in the liquidvapor surface; the Laplace pressure will be reduced towards zero. The vanishing contact angle is consistent with a vanishing pressure (here we are only concerned with classical capillarity and so ignore van der Waals forces) and film formation. Importantly this argument is consistent with conserving the fluid volume.

On a fiber, film formation is inhibited. A vanishing equilibrium contact angle is not sufficient for film formation. A thin sheathing film around a fiber must be consistent with fluid volume being conserved. However, if we spread the fluid along the fiber we necessarily reduce the radius of the film about the fiber axis. At the contact line one principal radius will increase whilst the other will decrease to maintain fluid volume. Physically, it seems clear that the way to minimize the Laplace excess pressure is to make one radius of curvature negative rather than positive at the contact line. Since the apex of the barrel must have both radii positive this means an inflexion angle must exist in the profile. For a vanishing equilibrium angle it is therefore possible to have a macroscopic barrel shape with a finite Laplace excess pressure. Thus, although the chemistry may determine the contact angle through Young's equation, the wetting of the fiber surface is strongly influenced by the global geometry of the cylindrical shape.

\section{PROFILE SOLUTION FOR A BARREL SHAPE}

The solution of Equation (1), subject to the boundary condition of a specific contact angle and axial symmetry, was given by Carroll (1976). This barrel-shape solution involves transforming the Laplace excess pressure using:

$$
R_{1} \mathrm{~d} \phi=\mathrm{d} x \sec \phi \quad \text { and: } \quad R_{2}=x \csc \phi
$$

and then imposing the boundary conditions:

$$
\begin{array}{ll}
\phi=\frac{\pi}{2} & \text { at } x=x_{2} \\
\phi=\frac{\pi}{2}-\theta_{e} & \text { at } x=x_{f}
\end{array}
$$

where $x_{f}$ is the fiber radius and $x_{2}$ is the maximum drop radius about the fiber. The solution is derived in terms of elliptic integrals and can be used to evaluate volume, pressure and surface areas, and hence the surface free energy (McHale et al., 1997). Measurement of drop shape parameters, such as wetted length and drop thickness, can then be used to estimate the contact angle (McHale et al., 1997, 1999; Carroll, 1976).

Re-examining the solution we can understand the link to the idea of a change in sign of one of the radii of curvatures at the contact line. Equation (2) is not simply a coordinate transformation, but involves a path in the $\phi$-parameter space. For any value of $x$, the angle $\phi$ is $90^{\circ}$ minus the slope of the profile. In principle, two possible paths in the $\phi$-parameter space exist as shown in Figure 2. In the first case the angle $\phi$ changes in a single-valued and monotonic manner from the first boundary condition (Eq. (3)) to the second (Eq. (4)). In the second case the angle $\phi$ is no longer single-valued, but starts at the first boundary condition $(E q$. (3)), reduces by a maximum angle of $\theta_{i}$ and then increases back again to reach the second boundary condition (Eq. (4)). Whilst the same endpoints are reached in both cases the effect in Case 2 of approaching the end-point from an anticlockwise direction results in a change of sign for $R_{2}$ compared to $R_{1}$. This change in sign was argued earlier as being necessary to minimize the Laplace excess pressure. Of course, these two paths do not correspond to the same volume of fluid, but it is the case that the elliptic solution for the barrel-shape profile appears to pick out the second path rather than the first. Physically, the second path corresponds to a profile that has an inflexion angle, $\theta_{i}$,

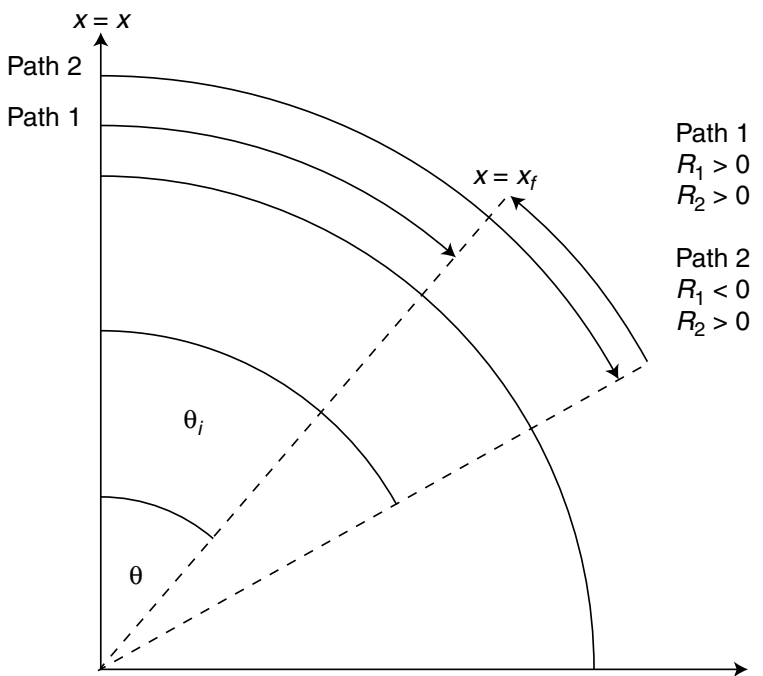

Figure 2

Two possible paths in the parameter space for the solution giving a barrel shape. The first path retains positive radii of curvature at all points whilst the second involves a change in relative sign between the two radii. 
with the contact angle occurring numerically once above this point and once below this point (i.e. at the contact line with the fiber).

\section{THE ROLL-UP TRANSITION}

Physically, a large volume droplet in a barrel shape without distortion due to gravity can be obtained using an oil drop on a fiber in an aqueous solution. Roll-up, the transition from a barrel shape to a clam-shell shape, can then be induced by solubilization of the oil drop using a surfactant. The drop volume then slowly reduces whilst maintaining an almost constant contact angle. Data from such experiments has been published in the literature and this is reproduced in Figure 3 together with a stability criterion suggested by Carroll (1986). This diagram shows the maximal drop thickness in reduced coordinates, $n=x_{2} / x_{f}$, and the contact angle, $\theta$, at which the barrel shape is lost and the clam-shell shape forms. From Figure 3 it is clear that the stability criterion given by the solid line mirrors the trend in the data. However, the agreement is far from satisfactory as the difference in maximal droplet thickness observed for a transition compared to the prediction can be more than a factor of two. At present, it is not clear why such a discrepancy should exist. The experiments themselves clearly have limited accuracy, as shown by the scatter in the data. This is not a surprise as the solubilization process is difficult to control and may involve nonequilibrium effects such as concentration and surface tension gradients. However, it is also possible that the stability condition itself may involve an invalid assumption.

To derive a stability condition for the barrel shape, Carroll (1986) first re-wrote the Laplace excess pressure in terms of the barrel parameters, $n$ and $\theta_{e}$ :

$$
\Delta P=\gamma_{L V}\left(\frac{1}{R_{1}}+\frac{1}{R_{2}}\right)=\frac{2 \gamma_{L V}}{x_{f}}\left(\frac{n-\cos \theta_{e}}{n^{2}-1}\right)
$$

He then argued that the two principal radii of curvature were therefore known at the apex of a drop (i.e. at $x=x_{2}$ ) since:

$$
\frac{1}{R_{2}}=\frac{x_{f}}{n}
$$

Equation (5) requires that the second radii of curvature be given by:

$$
\frac{1}{R_{2}}=\frac{1}{x_{f}}\left(\frac{n^{2}-2 n \cos \theta_{e}+1}{n\left(n^{2}-1\right)}\right)
$$

Carroll's argument was then to consider a perturbation of the shape (Fig. 4), using a change $\delta n$, whilst maintaining a constant volume. This type of perturbation will increase $R_{2}$

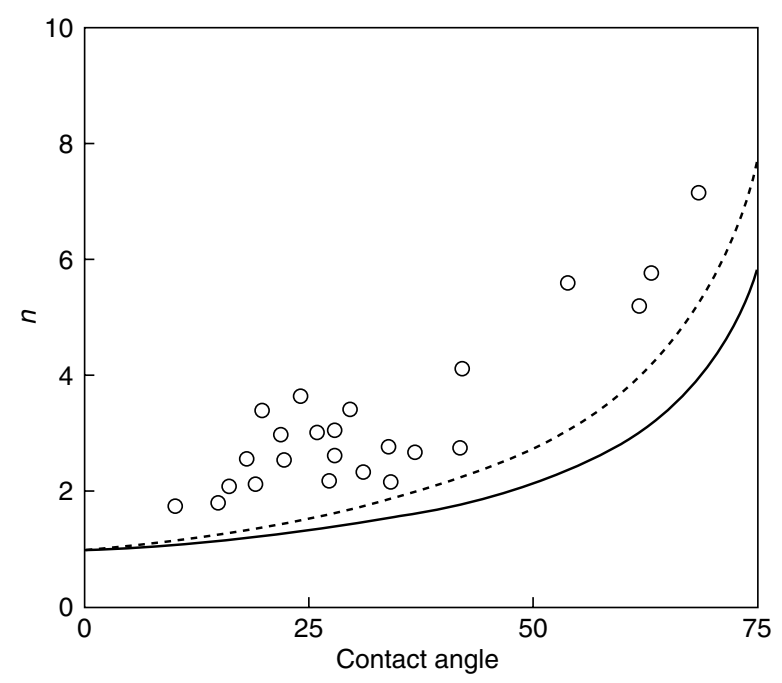

Figure 3

Literature data giving the contact angle and drop thickness at which a change of conformation of a drop on a fiber occurs (data is from Carroll, 1986). The solid line is Carroll's stability criterion and the dotted line shows the condition for the inflexion point to reach the contact line at the fiber surface.

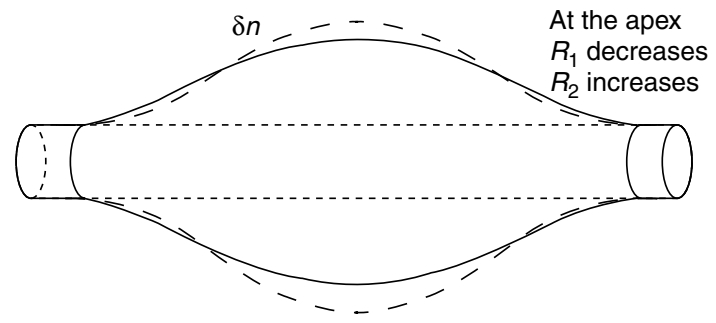

Figure 4

An initially barrel-shaped droplet (solid line) is perturbed by increasing its maximal thickness such that at the apex, $R_{2}$ increases and $R_{1}$ decreases (dashed line).

whilst decreasing $R_{1}$ and will therefore change the Laplace excess pressure calculated from Equation (1). The change in pressure due to this perturbation is:

$$
\delta(\Delta P)=-2 n \gamma_{L V}\left(\frac{2 n^{3} \cos \theta_{e}-3 n^{2}+1}{n^{2}\left(n^{2}-1\right)^{2}}\right)
$$

and this changes sign at specific values of $n$ and $\theta_{e}$. If we imagine the upper half of a drop increasing in volume and the lower half decreasing in volume, then for one sign of Equation (8) fluid will be driven back into the lower half thus restoring the shape, but for the other sign of pressure change fluid will be further driven into the top thus resulting in a destabilization of the barrel shape and the formation of a 
clam-shell shape. The stability condition given by the solid line in Figure 4 is therefore simply the condition for the sign change in Equation (8).

The stability argument is attractive, but there are several possible problems. The first is that an unphysical assumption may have implicitly occurred in the argument. A second possibility is that the condition does not refer to the absolute equilibrium as defined by a minimum in the surface free energy.

\section{CONJECTURE ON STABILITY}

Consider the second of the parameterized paths shown in Figure 2. This path describes a barrel shape with an inflexion angle in the profile. By moving the inflexion angle a perturbation of Path 2 would be possible whilst maintaining the equilibrium contact angle, and hence boundary conditions. For the barrel shape described by Path 2 , a perturbation of the inflexion angle would not alter the relative signs between the two principal radii of curvature at the contact line. However, as the volume of a barrel-shaped drop reduces, the inflexion angle also reduces towards the value of the contact angle. Eventually Path 2 apparently becomes the same as Path 1, but in the limit there is a difference between approaching the end-point of the path from the anticlockwise and the clockwise directions. In the former case, the radii of curvature have opposite signs whilst in the latter case the signs are the same. Changing from Path 2 to Path 1 involves a singularity. For Path 1, we no longer have an inflexion point on the physical profile of the drop to perturb. This may indicate that the stability argument is correct in predicting stability, but only in the restricted range of the parameter space corresponding to Path 2. Whilst it is not clear that this is indeed a flaw in the stability argument its consequences, should it be true, can be evaluated.

The condition for the angle of inflexion to become the same as the contact angle is known (McHale et al., 1997) and depends upon the contact angle and the volume (through the drop thickness):

$$
n_{\min }=\frac{1+\sin \theta_{e}}{\cos \theta_{e}}
$$

Equation (9) is plotted as the dotted curve in Figure 3. The trend of the curve again mirrors that of the experimental data. Exact numerical agreement is not obtained, but it is closer than the previous stability condition (solid curve). All points above the dotted curve lie on Path 2 and correspond to barrel shapes with inflexions in their profiles. According to Carroll's stability argument these points correspond to barrel shapes that are also stable against perturbations in their profiles. Points in Figure 3 that are between the solid and the dotted curves correspond to hypothetical barrel shapes using Path 1 and they have therefore no inflexion in their profiles.
In this approach, the question of stability is therefore converted into a question of whether such barrel shapes can exist. We offer no definite answer to this question. The conjecture for stability, and hence the roll-up transition, can be phrased as the following, stability of a barrel shape is lost when the reduction in volume causes the inflexion point to touch the fiber surface. Mathematically, this conjecture is given by Equation (9).

\section{SURFACE FREE ENERGY CONSIDERATIONS}

The surface free energy of a droplet on solid system can be calculated from the interfacial areas, provided the shape is known. For the axisymmetric barrel shape the profile solution is known in terms of elliptic integrals and surface free energy calculations are possible (McHale et al., 1997). A similar calculation cannot be performed for a clam-shellshaped drop as no solution to this non-axisymmetric shape is known. To determine which of the two conformations is energetically preferred it is necessary to calculate the equilibrium shape of both droplets. The completion of such calculations would enable the condition for absolute stability to be determined, at least numerically. We have therefore begun preliminary calculations of the equilibrium shape of clam-shell type droplets subject to a specified fluid volume and equilibrium contact angle. The numerical approach is to apply finite element computations using the public domain Surface Evolver package (Brakke, 2000), which was developed as part of an NSF (National Science Foundation) funded geometry supercomputing project. In this method surfaces are modeled as unions of triangles and vertices are iteratively moved from an initial trial shape until a minimum energy configuration is obtained. In our case, the energy is given solely by surface tension and constraints are imposed on the volume, contact angle and the cylindrical shape of the solid surface. Surface Evolver is particularly flexible and allows energies and coordinate profiles to be extracted from the evolved shape. However, movement of vertices, edges and facets on constrained faces can be problematic. The energy of a facet on a constrained face is therefore converted to a line integral around the boundary. One potential problem for small diameter fibers is that spurious reductions in energy can be generated by lines short-circuiting the fiber crosssection, although we did not find this to be a problem.

Figure 5 shows the starting shape and the finite-elementevolved shapes for four barrel type droplets (Figs. 5a-5d). The first two of these droplets correspond to an equilibrium contact angle of $0^{\circ}$ and reduced volumes (i.e. volume $/ x_{f}^{3}$ ) of 208.8 and 53.75 respectively. The second uses a higher contact angle of $60^{\circ}$, but with the same reduced volumes. Using 1056 vertices, the surface free energies normalized by $\gamma_{L V}$ predicted from these shapes are 122.3, 40.8, 152.6 and 60.09. These results are close to the values of $122.2,40.5$, 152.6 and 60.08 obtained by using the elliptic integral 

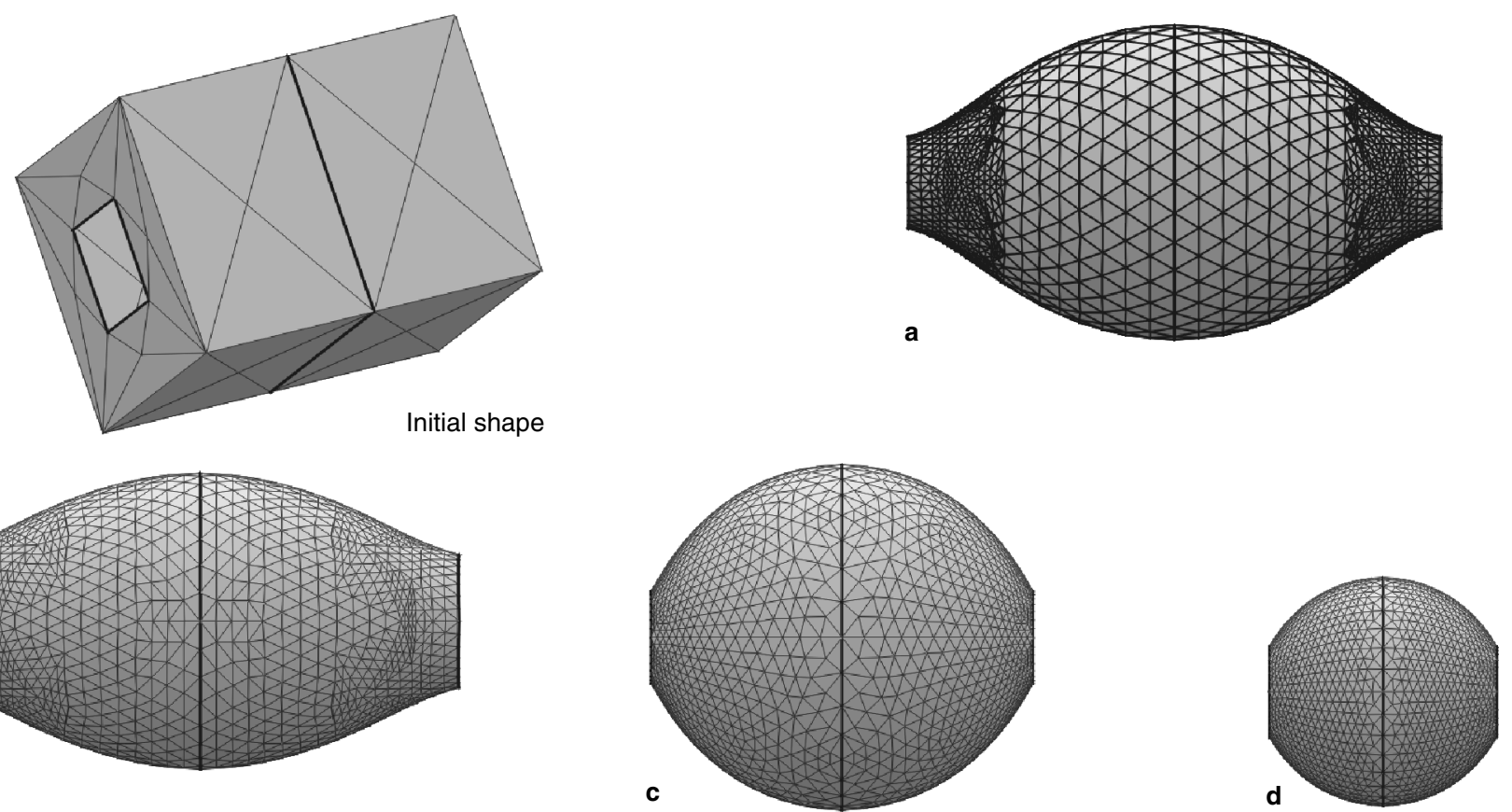

Figure 5

Finite element starting approximation for an axisymmetric drop. Finite-element-evolved shapes for contact angles and reduced volumes of (a) $0^{\circ}$ and 208.8, (b) $0^{\circ}$ and 53.75, (c) $60^{\circ}$ and 208.8 and (d) $60^{\circ}$ and 53.75 .

solutions for the profiles ${ }^{1}$; Figures 5-7 are plotted using less vertices. The good agreement for the surface free energy between the two numerical approaches provides confidence that the finite element method is working correctly. Figures 6 and 7 show the finite element calculations using the same liquid volumes and contact angles, but with a starting shape that is appropriate to a non-axisymmetric solution. Figure $6 \mathrm{a}$ shows a view down the axis of the fiber for the large droplet with a vanishing contact angle. This shape does not evolve to a clam-shell equilibrium because further iterations lead to the lower part of the shape joining; physically this corresponds to the formation of a barrel shape. Figure $6 \mathrm{~b}$ shows the axial and side views of the smaller droplet with a zero contact angle. In this case the lower parts have not joined, but the surface free energy of 42.12 is larger than that for the barrel shape (Fig. 5b). The angular shape of lower part of the side view indicates that further improvements in the finite element shape can be expected with an increase in the number of vertices. For the larger contact angle of $60^{\circ}$, shown in Figures $7 \mathrm{a}$ and $7 \mathrm{~b}$, we find the large volume drop evolves to a shape where the two sides of the drop join in the same manner as in Figure $6 \mathrm{a}$. In the last case of a contact angle of $60^{\circ}$ and a reduced volume of 53.75 (Fig. $7 \mathrm{~b}$ ) the shape evolves to a clam-shell type shape similar to Figure 6b. However, in this

(1) A number of programs for calculating the contact angle and surface free energy for a barrel shape from measurements of barrel parameters are available from the corresponding author.
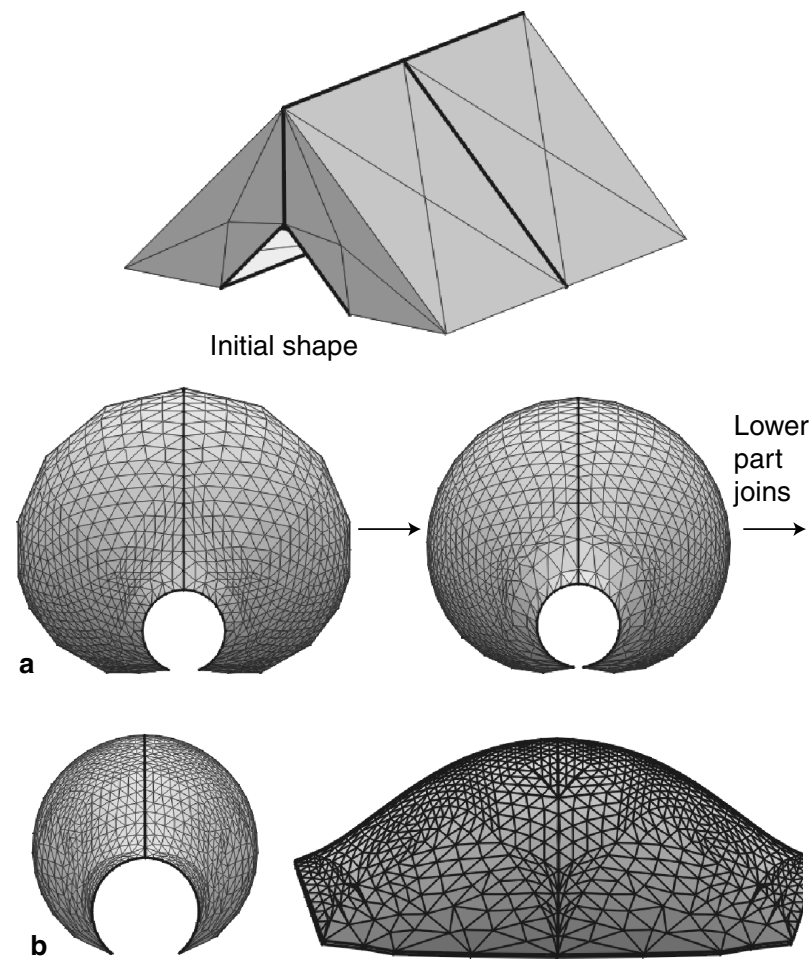

Figure 6

Finite element starting approximation for a clam-shell-shaped drop. Finite-element-evolved shapes for a contact angle of $0^{\circ}$ and reduced volumes of (a) 208.8 and (b) 53.75 (axial and side views). 

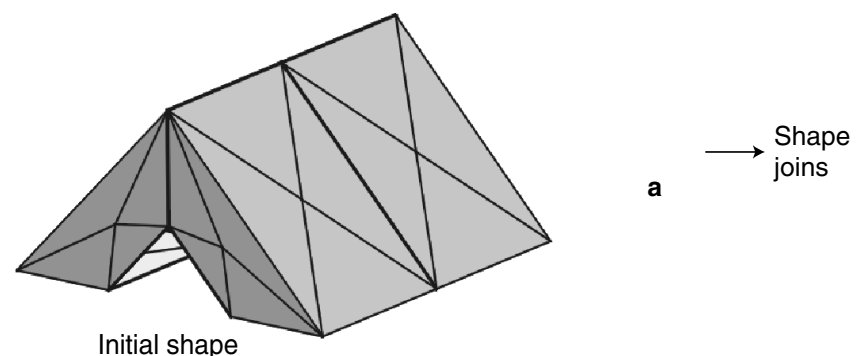

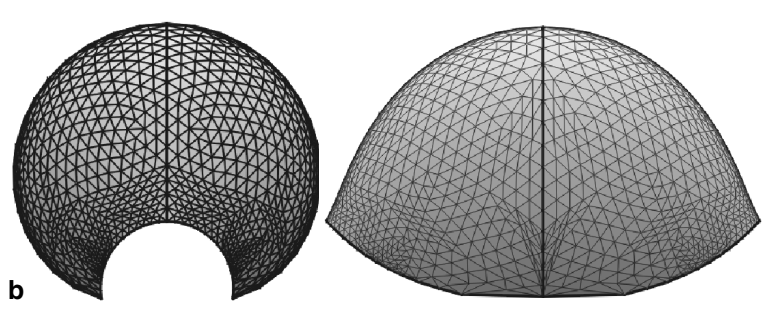

Figure 7

Finite element starting approximation for a clam-shell-shaped drop. Finite element results for a contact angle of $60^{\circ}$ and reduced volumes of (a) 208.8 and (b) 53.75 (axial and side views).

case the predicted energy is 56.75 , which is less than for the equivalent barrel shape. Refinements of the finite element mesh would be expected to further reduce the predicted energy and would therefore confirm that the clam-shell is the preferred conformation for the parameters in Figure 5c.

These finite element calculations are only preliminary, but they suggest that it will be possible to determine numerically the shape of clam-shell drops and their surface free energy. The construction of a diagram showing absolute stability should then be possible. However, absolute stability may not be appropriate for describing the roll-up transition. The finite element approach should allow a direct test of the stability of an equilibrium clam-shell-shaped droplet by numerically perturbing the coordinates of the vertices. Further evolving of the perturbed shape may then lead to either a barrel shape or, alternatively, restore the clam-shell shape. One limitation of the finite element approach and the previous analytical stability arguments in this work is that they rely purely on classical capillary considerations and ignore van der Waals forces and microscopic films that can exist in practice. To overcome these limitations, numerical simulations of wetting on fibers, using for example molecular dynamics as previously applied to wetting on flat surfaces (de Ruijter $e t$ al., 1999), or numerical evaluation of free energy functionals (Bauer and Dietrich, 2000), could be used to provide an alternative approach to the assessment of the stability of the conformations and the roll-up process.

\section{CONCLUSION}

The global geometric shape of a solid surface as well as the surface chemistry can substantially alter the wetting properties of the surface. On a fiber this is apparent from the behavior of drops with a vanishing equilibrium contact angle, the formation of films and the existence of two conformations of droplets. Stability of a barrel-shape drop may be perturbed by reducing the drop volume or increasing the contact angle and this is described as the roll-up process.
The validity of the existing criteria for roll-up has been considered and it has been conjectured that stability may be lost when the drop volume is reduced to such an extent that the inflexion in the profile of the drop reaches the fiber surface. A finite-element-based approach to examining the absolute stability of the two conformations based on minimum surface free energy has been outlined. Initial results are promising and suggest both clam-shell profiles and the absolute stability diagram can be constructed.

\section{REFERENCES}

Adam, N.K. (1937) J. Soc. Dyers Colour, 53, 122-128.

Adamson, A.W. and Gast, A.P. (1997) Physical Chemistry of Surfaces, John Wiley \& Sons, New York.

Bauer, C. and Dietrich, S. (2000) Shapes, Contact Angles and Line Tensions of Droplets on Cylinders. Report WUB 00-0, Bergische Universität Wuppertal, Germany.

Brakke, K.A. (2000) Surface Evolver. www.susqu.edu/facstaff/b/brakke/evolver.

Brochard, F. (1986) Spreading of Liquid-Drops on Thin Cylinders. The Manchon-Droplet Transition. J. Chem. Phys., 84, 4664-4672.

Brochard-Wyart, F., Di Meglio, J.M. and Quéré, D. (1990) Theory of the Dynamics of Spreading of Liquids on Fibers. J. Phys. Paris, 51, 293-306.

Carroll, B.J. (1976) The Accurate Measurement of Contact Angle, Phase Contact Areas, Drop Volume, and Laplace Excess Pressure in Drop-on-Fiber Systems. J. Colloid Interface Sci., 57, 488-495.

Carroll, B.J. (1986) Equilibrium Conformations of Liquid-Drops on Thin Cylinders under Forces of Capillarity-A Theory for the Roll-up Process. Langmuir, 2, 248-250.

Carroll, B.J. (1993) Physical Aspects of Detergency. Colloid Surface, A74, 131-167.

De Gennes, P.G. (1985) Wetting: Statics and Dynamics. Rev. Mod. Phys., 57, 827-863.

De Ruijter, M.J., Blake, T.D. and De Coninck, J. (1999) Dynamic Wetting Studied by Molecular Modeling Simulations of Droplet Spreading. Langmuir, 15, 7836-7847. 
Léger, L. and Joanny, J.F. (1992) Liquid Spreading. Rep. Prog. Phys., 55, 431-486.

McHale, G., Käb, N.A., Newton, M.I. and Rowan, S.M. (1997) Wetting of a High-Energy Fiber Surface. J. Colloid Interface Sci., 186, 453-461.

McHale, G., Rowan, S.M., Newton, M.I. and Käb, N.A. (1999) Estimation of Contact Angles on Fibers. J. Adhesion Sci. Technol., 13, 1457-1469.
Quéré, D. (1999) Fluid Coating on a Fiber. Ann. Rev. Fluid Mech., 31, 347-384.

Richard, D. and Quéré, D. (1999) Viscous Drops Rolling on a Tilted Non-Wettable Solid. Europhys. Lett., 48, 286-291.

Wenzel, R.N. (1936) Ind. Eng. Chem., 28, 988.

Final manuscript received in November 2000 